\title{
Health Insurance Gains After Implementation of the Affordable Care Act Among Individuals Recently on Probation: USA, 2008-2016
}

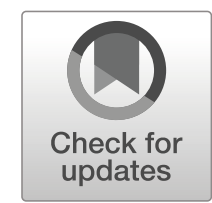

\author{
Christopher D. Knapp, MD, MPH ${ }^{7}$, Benjamin A. Howell, MD, MPH ${ }^{2}$, Emily A. Wang, MD, MAS $S^{2,3}$, \\ Rebecca J. Shlafer, $P h D, M P H^{4}$, Rachel R. Hardeman, PhD, MPH ${ }^{5}$, and Tyler N. A. Winkelman, \\ $M D, M S c^{6,7}$
}

\begin{abstract}
'Internal Medicine Residency Program, Hennepin Healthcare, Minneapolis, MN, USA; ${ }^{2}$ National Clinician Scholars Program, Yale School of Medicine, New Haven, CT, USA; ${ }^{3}$ General Internal Medicine, Yale School of Medicine, New Haven, CT, USA; ${ }^{4}$ Department of Pediatrics, University of Minnesota Medical School, Minneapolis, MN, USA; ${ }^{5}$ Division of Health Policy and Management, School of Public Health, Minneapolis, MN, USA;

${ }^{6}$ General Internal Medicine, Hennepin Healthcare, Minneapolis, MN, USA; ${ }^{7}$, Hennepin Healthcare Research Institute, Minneapolis, MN, USA.
\end{abstract}

J Gen Intern Med 34(7):1086-8

DOI: $10.1007 / \mathrm{s} 11606-019-04900-3$

(c) Society of General Internal Medicine 2019

\section{INTRODUCTION}

Over 6 million adults are supervised by the US correctional system on any given day. ${ }^{1}$ Less than half, 2.2 million, are incarcerated in jails or prisons, while 3.7 million are on probation and serve their sentences while living in the community. ${ }^{1}$ People involved in the correctional system are more likely to have a chronic condition, substance use disorders, or mental illness and are at higher risk of being uninsured when compared with the general population. ${ }^{2-5}$

In the first year after implementation of the Affordable Care Act's (ACA) key provisions, health insurance coverage increased for individuals with involvement in the criminal justice system. ${ }^{2,6}$ Whether the ACA continued to improve coverage beyond its first year of implementation among this population is unknown. Furthermore, no analyses have disaggregated the impact of the law for individuals with probation. It is critical to examine the impact of the ACA for individuals with probation, because they are a high-risk population that is community-dwelling and can use health insurance to engage with the traditional health care system.

To assess the three-year impact of the ACA among US adults with recent probation, we examined changes in health insurance coverage between 2008 and 2016.

\section{METHODS}

We analyzed data from the 2008-2016 National Survey of Drug Use and Health, ${ }^{7}$ a nationally representative crosssectional survey that measures the prevalence of mental health and substance use in the USA. ${ }^{7}$ Our study population was restricted to non-elderly adults aged 18-64. Individuals on probation were identified as such if they reported being on probation in the past year.

Our primary outcome variable was insurance status. An individual was considered to be insured if they reported any private insurance, Medicare, Medicaid/Children's Health Insurance Program, military-related insurance, or other health insurance. Individuals with any private insurance were labeled private, those with Medicaid but not private insurance were labeled Medicaid, and those with any other insurance were labeled other.

We estimated insurance coverage during each year of our study for individuals with and without probation using logistic regression and predictive margins. We used logistic regression models and predictive margins, controlling for age, sex, education level, race/ethnicity, income, marital status, and employment status to obtain a difference-in-differences (DID) estimate of the ACA's impact among individuals with probation in the past year, relative to the general population, from 2008-2013 to 2015-2016. We excluded data from 2014 as a transition period due to implementation of the marketplaces and Medicaid expansion that year.

Unadjusted frequencies were used to describe health insurance categories for individuals on probation from 2013 to 2016. Significance testing was conducted using logistic regression.

Analyses were conducted with Stata/MP, version 15.1 (Stata Corp), and accounted for the NSDUH's complex survey design.

\section{RESULTS}

Our unweighted sample included 11,511 individuals with probation and 319,652 without probation in the past year. In weighted analyses, 4,755,829 individuals, on average,

Published online February 27, 2019 


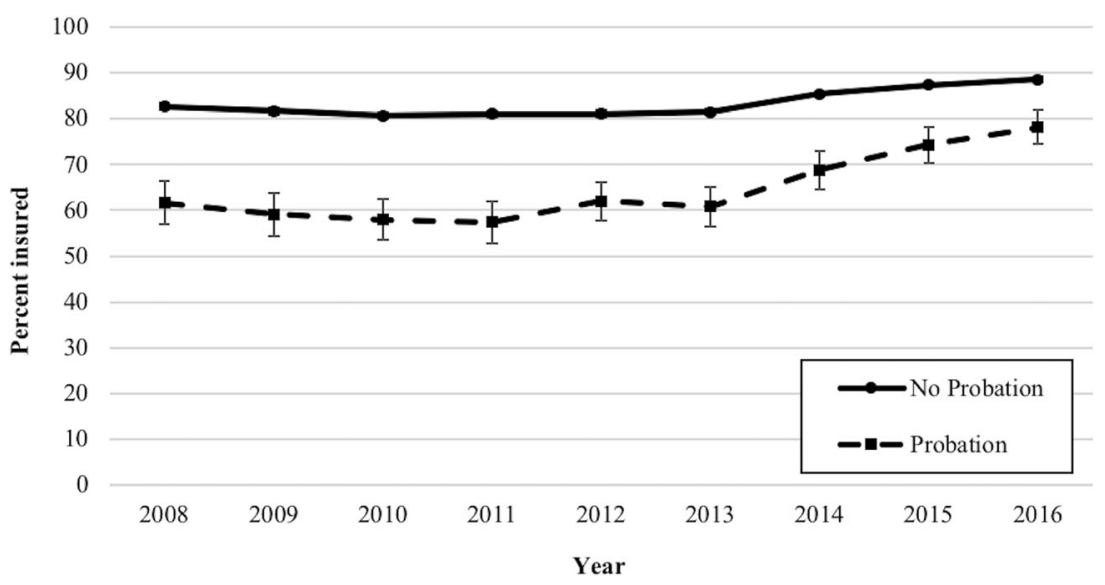

Fig. 1 Health insurance coverage among individuals with and without a history of probation in the past year-USA, 2008-2016.

reported probation annually. Individuals with probation were significantly more likely to be male $(70.5 \%$ vs. $48.5 \%$; $P<.001)$ and Black, non-Hispanic $(18.2 \%$ vs. $12.1 \%$; $P<.001)$ compared with the general population.

Insurance coverage increased significantly between 2013 and 2016 for individuals with probation (17.4 percentage points [95\% CI, 11.8-23.0]) (Fig. 1), but remained lower compared with that for the general population. After adjustment, insurance coverage increased significantly more among individuals who reported probation in the past year than in the general population $(10.6 \%$ vs. $6.7 \% ; P<.001)$.

Among individuals with probation in the past year, private health insurance and other insurance did not increase significantly between 2013 and 2016, whereas Medicaid coverage increased significantly (18.7 to $31.3 \%$; $P=<.001$ ) (Fig. 2).

\section{DISCUSSION}

Following implementation of the ACA's key provisions in 2014, insurance coverage increased significantly for individuals on probation. Although insurance coverage remained lower for individuals with probation compared with that for individuals without probation, coverage disparities decreased significantly between 2013 and 2016 . Health insurance gains among individuals on probation in the past year were primarily due to an increase in Medicaid coverage. Our results highlight Medicaid expansion's role in reducing disparities in health insurance coverage for this marginalized population. Future work should examine whether insurance gains among individuals with probation led to changes in health care use or better health outcomes.

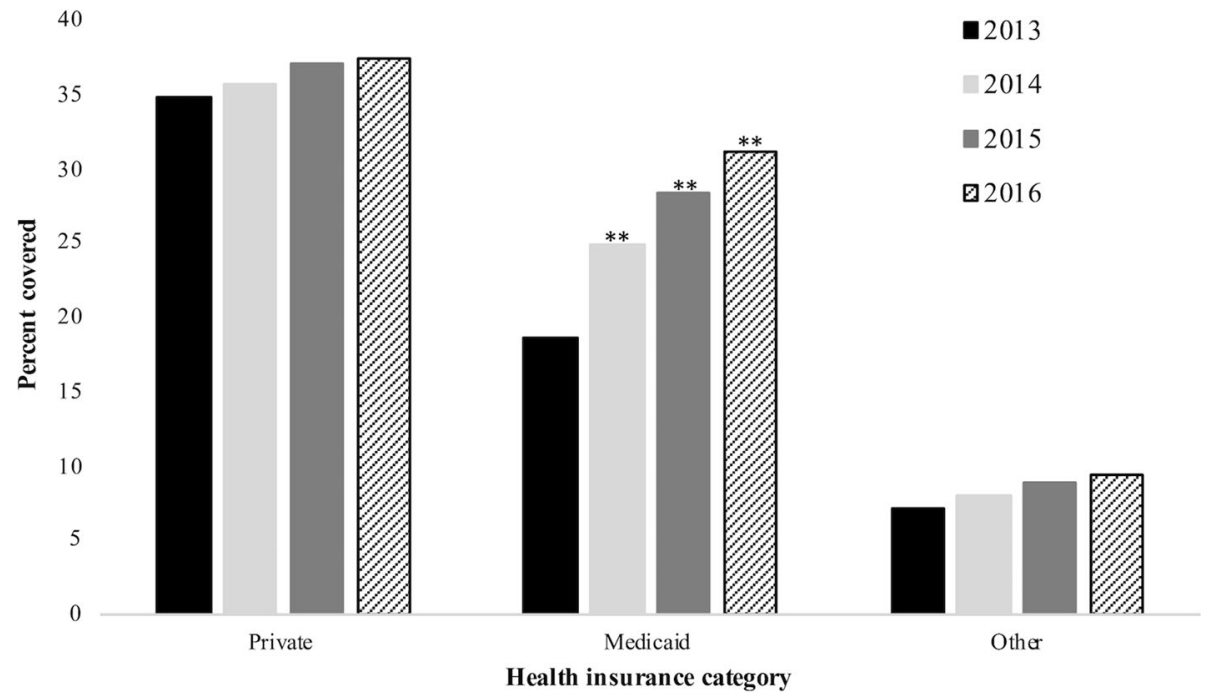

Fig. 2 Types of health insurance coverage among individuals with a history of probation in the past year-USA, 2013-2016. $* * P<.05$ compared to 2013 . 
Corresponding Author: Christopher D. Knapp, MD, MPH; Internal Medicine Residency Program, Hennepin Healthcare, 701 Park Ave, Medicine Education Program, G5, Minneapolis, MN 55415, USA (e-mail: christopher.knapp@hcmed.org).

Funding Information Dr. Winkelman received funding through a career development award from Hennepin Healthcare. Drs. Winkelman, Shlafer, and Hardeman received funding through the University of Minnesota's Grand Challenges Initiative.

\section{Compliance with Ethical Standards:}

Conflict of Interest: The authors declare that they do not have a conflict of interest.

Publisher's Note: Springer Nature remains neutral with regard to jurisdictional claims in published maps and institutional affiliations.

\section{REFERENCES}

1. Kaeble D, Cowhig M. Correctional Populations in the United States, 2016. Washington, DC: Bureau of Justice Statistics; 2018. https://www.bjs.gov/ content/pub/pdf/cpus 16.pdf. Accessed December 17th, 2018.

2. Winkelman TNA, Kieffer EC, Goold SD, Morenoff JD, Cross K, Ayanian JZ. Health Insurance Trends and Access to Behavioral Healthcare Among
Justice-Involved Individuals-United States, 2008-2014. J Gen Intern Med. 2016;31(12):1523-1529. doi:https://doi.org/10.1007/s11606-0163845-5

3. Winkelman TNA, Chang Vw, Binswanger IA. Health, polysubstance use, and criminal justice involvement among adults with varying levels of opioid use. JAMA Netw Open. 2018;1(3):e180558. doi:https://doi.org/10.1001/ jamanetworkopen.2018.0558

4. Fazel S, Hayes AJ, Bartellas K, Clerici M, Trestman R. Mental health of prisoners: prevalence, adverse outcomes, and interventions. Lancet Psychiatry. 2016;3(9):871-881. doi:https://doi.org/10.1016/S2215-0366(16) 30142-0

5. Binswanger IA, Krueger PM, Steiner JF. Prevalence of chronic medical conditions among jail and prison inmates in the USA compared with the general population. J Epidemiol Community Health. 2009;63(11):912919. doi:https://doi.org/10.1136/jech.2009.090662

6. Saloner B, Bandara SN, McGinty EE, Barry CL. Justice-Involved Adults With Substance Use Disorders: Coverage Increased But Rates Of Treatment Did Not In 2014. Health Aff (Millwood). 2016;35(6):1058-1066. doi:https://doi.org/10.1377/hlthaff.2016.0005

7. National Survey on Drug Use and Health, 2017. Washington, D.C.: United States Department of Health and Human Services. Substance Abuse and Mental Health Services Administration. Center for Behavioral Health Statistics and Quality; 2017. https://www.samhsa.gov/data/sites/default/files/NSDUH-DetTabs-2016/NSDUH-DetTabs-2016.htm. Accessed 20 Feb 2019. 